INPLASY

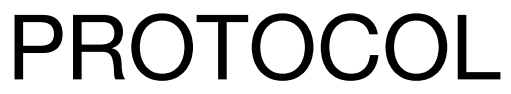

To cite: Tu. A systematic review and meta-analysis : the external therapeutic effect of traditional Chinese medicine external therapy on rat knee osteoarthritis. Inplasy protocol 2020110103. doi:

10.37766/inplasy2020.11.0103

Received: 22 November 2020

Published: 23 November 2020

Corresponding author:

Shijuan Tu

1148217370@qq.com

Author Affiliation:

Chengdu University of

Traditional Chinese Medicine

Support: None.

Review Stage at time of this submission: Preliminary searches.

Conflicts of interest:

None.

\section{A systematic review and meta- analysis : the external therapeutic effect of traditional Chinese medicine external therapy on rat knee osteoarthritis}

Tu, S1.

Review question / Objective: To evaluate the efficacy of the external therapeutic effect of Traditional Chinese medicine therapy on rat knee osteoarthritis.

Condition being studied: Therapy of traditional Chinese medicine; muroid; knee osteoarthritis.

Information sources: The details were adjusted according to the specific databases including CBM, CNKI, WF, VIP, Web of Science, Embase, PubMed, Cochrane Library, WHO ICTRP, ChiCTR, Clinical Trials, Grey Literature Database. No limitation on language or publication types restriction will be applied.

INPLASY registration number: This protocol was registered with the International Platform of Registered Systematic Review and Meta-Analysis Protocols (INPLASY) on 23 November 2020 and was last updated on 23 November 2020 (registration number INPLASY2020110103).

\section{INTRODUCTION}

Review question / Objective: To evaluate the efficacy of the external therapeutic effect of Traditional Chinese medicine therapy on rat knee osteoarthritis.
Condition being studied: therapy of traditional Chinese medicine; muroid; knee osteoarthritis.

\section{METHODS}

Search strategy: The details were adjusted according to the specific databases including Chinese Biomedical Literature 
(CBM), the China National Knowledge Infrastructure Database (CNKI), Wangfang Database (WF), Chinese Scientific Journal Database (VIP), Web of Science, Embase, PubMed, Cochrane Library, the World Health Organzation International Trials Registry Platform (WHO ICTRP), Chinese Clinical Trial Register (ChiCTR), Clinical Trials, Grey Literature Database. No limitation on language or publication type's restriction will be applied.

Participant or population: Inclusion: Different types of mice with knee osteoarthritis could be included in the study, regardless of the type, weight, sex, or mode of modeling of the mice.Exclusion: Model mice with rheumatoid arthritis, infectious arthritis, and hormone-induced arthritis were excluded.

Intervention: The focus of this study is the animal experiment on the treatment of rat knee osteoarthritis by TCM external therapy. The results will provide Suggestions for researchers to study the mechanism of KOA occurrence and development.Therefore, the experimental group only accepted external TCM therapy, and did not accept any combination of other Western medicine, internal TCM therapy and therapy, regardless of the type, duration and frequency of external therapy.Animal experiments that cannot evaluate the efficacy of TCM external therapies will be excluded.

Comparator: The control group will be treated with western medicine, traditional Chinese medicine and other combinations of treatment.

Study designs to be included: A Chinese English randomized controlled trial of rat knee joint osteoarthritis externally treated with Traditional Chinese medicine was included. Non-randomized controlled trials, reviews, case reports, experimental studies, expert experience, and republished papers were excluded.

Eligibility criteria: A Chinese - English randomized controlled trial of rat knee joint osteoarthritis externally treated with Traditional Chinese medicine was included.

Information sources: The details were adjusted according to the specific databases including CBM, CNKI, WF, VIP, Web of Science, Embase, PubMed, Cochrane Library, WHO ICTRP, ChiCTR, Clinical Trials, Grey Literature Database. No limitation on language or publication types restriction will be applied.

Main outcome(s): Articular cartilage damage will be evaluated as a primary outcome.The modified Mankin's scoring criteria (structure of each layer of cartilage, chondrocytes, HE staining of each layer of cartilage matrix, and tidal line integrity) were used to evaluate the degree of cartilage degeneration.

Quality assessment / Risk of bias analysis: Literature retrieval, screening and data extraction were conducted by two researchers independently through a standardized eligibility form. In case of disagreement, a third party shall be consulted to assist judgment, and the missing information shall be supplemented by contacting the author. The general information of the selected articles will be extracted, including first author, country, year of publication, study design, duration of follow-up, duration of disease, sample size, detailed intervention, control treatment and the like. When the data of articles are sufficient or ambiguous, one of the authors will contact the original author to request detailed and additional information by e-mail or telephone.

Strategy of data synthesis: RevMan5.3.5 will be used for all statistical analyses. Based on the heterogeneity levels of the included studies, the fixed-effects model (I2 $250 \%)$ or random-effects model (I2 $250 \%)$ will be selected. The dichotomous data will be analyzed by RR with $95 \%$ Cls, while the continuous data will be analyzed by MD/ SMD with $95 \%$ Cls. The meaningful heterogeneity will be explained by any additional assessment included sensitivity analysis or subgroup analysis depended on the data. 
Subgroup analysis: If necessary, Subgroup analysis will be performed based on different types of acupuncture therapy, participant characteristics, and outcome measures.

Sensibility analysis: When the subgroup analysis is unsatisfactory, we will use sensitivity analysis to evaluate the robustness of the main results. Then metaanalysis reorganizes and merges the data, and finally compares with the previous results.

Country(ies) involved: China.

Keywords: External therapy of traditional Chinese medicine; muroid; knee osteoarthritis; systematic review.

Contributions of each author:

Author 1 - Shijuan Tu. 